\title{
Human neutrophil peptides $1-3$ as gastric cancer tissue markers measured by MALDI-imaging mass spectrometry: Implications for infiltrated neutrophils as a tumor target
}

\author{
Chun-Chia Cheng ${ }^{\mathrm{a}, \mathrm{b}}$, Jungshan Chang ${ }^{\mathrm{a}, \mathrm{d}, \mathrm{e}, \mathrm{f}}$, Ling-Yun Chen ${ }^{\mathrm{g}}$, Ai-Sheng Ho ${ }^{\mathrm{h}}$, Ker-Jer Huang ${ }^{\mathrm{i}}$, \\ Shui-Cheng Lee ${ }^{\mathrm{b}}$, Fu-Der Mai ${ }^{\mathrm{a}, \mathrm{c}, \mathrm{d}, \text {, and Chun-Chao Chang }}{ }^{\mathrm{j}, *}$ \\ ${ }^{a}$ Graduate Institute of Medical Sciences, College of Medicine, Taipei Medical University, Taipei, Taiwan \\ ${ }^{\mathrm{b}}$ Institute of Nuclear Energy Research, Atomic Energy Council, Taoyuan, Taiwan \\ ${ }^{\mathrm{c}}$ Department of Biochemistry, School of Medicine, Taipei Medical University, Taipei, Taiwan \\ ${ }^{\mathrm{d}}$ Biomedical Mass Imaging Research Center, Taipei Medical University, Taipei, Taiwan \\ ${ }^{\mathrm{e}}$ Neuroscience Research Center, Taipei Medical University Hospital, Taipei, Taiwan \\ ${ }^{\mathrm{f}}$ Research Center for Biomedical Implants and Microsurgery Devices, Taipei Medical University, Taipei, Taiwan \\ ${ }^{\mathrm{g}}$ Institute of Biochemistry and Biotechnology, Chung Shan Medical University, Taichung, Taiwan \\ ${ }^{\mathrm{h}}$ Division of Gastroenterology, Cheng Hsin General Hospital, Taipei, Taiwan \\ ${ }^{\mathrm{i}}$ Chung-Shan Institute of Science \& Technology Armaments Bureau, Taoyuan, Taiwan \\ ${ }^{j}$ Department of Internal Medicine, School of Medicine, College of Medicine, Taipei Medical University Hospital, \\ Taipei, Taiwan
}

\begin{abstract}
Objective: Human neutrophil peptides (HNPs) -1, -2 and -3 are significantly upregulated and were reported as biomarkers in gastric cancer (GC). However, the tissue location and function of HNPs 1-3 are still unclear in GC, and the spatial distribution of the triad needs to be disclosed. The aims of this study were to investigate the distribution and relationships among HNPs-1, -2 and -3 , and assess whether infiltrated neutrophils accumulate in gastric tumor.

Methods: In this study, paired samples $(n=33)$ of the GC tissues and adjacent normal tissues from the same patients were obtained from surgery. Expression of HNPs 1-3 were detected by matrix-assisted laser desorption ionization time-of-flight mass spectrometry (MALDI-TOF MS). The distributions of the HNPs 1-3 in GC tissues were investigated. After verification of HNPs-1 by immunohistochemistry, infiltrated neutrophils were also detected. Then, an in vitro assay was used to observe the binding capacity and measure the cytotoxic effect of HNPs-1 against AGS cells.

Results: Comparing to neighboring normal tissue, expressional level of HNPs 1-3 were significantly higher and their distributions overlapped in cancerous tissues of GC patients with high abundance in the lamina propria, whereas HNPs-1 was identified as the highest major peak. Moreover, HNPs-1, -2 and -3 correlated with each other. Besides, we also observed that increased infiltrated neutrophils accumulating in GC tissues, indicating that a strong positive correlation between HNPs 1-3 and infiltrated neutrophils. In addition, the further investigated demonstrated that the major peptide, HNPs-1, was statistically increased with the advance of tumor development from the early to advanced stage of GC $(p<0.05)$. Moreover, we also noticed that HNPs- 1 with a great binding capacity to GC AGS cells in vitro can inhibit tumor cell growth.
\end{abstract}

Conclusions: Our results suggest that neutrophil secreted peptides, HNPs 1-3, increased in the GC tissues and could be used as

\footnotetext{
* Corresponding author: Chun-Chao Chang, Division of Gastroenterology, Taipei Medical University Hospital, 250, Wu Hsing Street,
}

Taipei 110, Taiwan. Tel.: +886227372181 ext. 3903; Fax: +8862 27363051; E-mail: chunchao@tmu.edu.tw. 
potential biomarkers detected using MALDI-TOF MS, implying that elevated neutrophils may be used as a tumor target for tumor treatment. The binding capacity of HNPs-1 with GC cells implies that tracking molecules conjugated with HNPs- 1 could be applied as a specific probe for GC diagnoses.

Keywords: Gastric cancer, human neutrophil peptides 1-3, MALDI-TOF MS, biomarker

\section{Introduction}

Gastric cancer (GC), mostly diagnosed as gastric adenocarcinoma, is a malignant gastric epithelial tumor that originates from the glandular epithelium of the gastric mucosa. According to the current statistic data, GC is the fourth most common cancer and the second most common mortal malignancy, which causes approximately 800,000 deaths annually worldwide, especially in Asia [1-4]. Early detection of GC is considered helpful for tumor treatment; thus, searching for and defining reliable biomarkers of GC coupled with improved and precise diagnostic methods are urgently needed to prevent further metastasis and enhance therapeutic success.

Gastroscopy followed by a histological examination of a biopsy specimen is still the gold standard for diagnosing GC in the clinic. However, it is invasive, time consuming, expensive, and physically unpleasant for most patients. Moreover, missed detection still commonly occurs [5]. Human neutrophil peptides (HNPs) alpha defensin 1,2, and 3, packaged in neutrophil granules, are reported to be especially highly expressed in a variety of cancers, including metastatic colorectal cancer [6,7], bladder cancer [8], renal cell carcinoma [9], squamous cell carcinoma [10], and breast cancer [11]. The characteristics of HNPs 1-3 in cancer tissues may be useful and applied for tumor diagnosis or therapy. However, the spatial locations and functions of HNPs$1,-2$ and -3 still remain unknown in GC.

In the previous studies, it has been demonstrated that HNPs 1, 2 and 3 display an ambiguous biofunctions toward the survival of tumor cells. Under lower concentration in the growth medium, HNPs 13 can promote tumor cell growth; in contrast, however, they possess cytotoxicity to tumor in higher concentration [12]. Therefore, it is important to disclose the roles of HNPs 1-3 to understand their putative functions correlated with GC. Previously, Kon's group indicated that HNPs 1-3 are significantly upregulated as biomarkers of GC as discovered in gastric fluid [13]. Other studies also indicated that HNPs 13 have the potential role to serve as biomarkers for $\mathrm{GC}$, as they are expressed in cancerous tissues using surface-enhanced laser desorption/ionization time-of- flight mass spectrometry (SELDI-TOF-MS) or matrixassisted laser desorption ionization time-of-flight mass spectrometry (MALDI-TOF MS) [14,15]. However, the spatial distributions of HNPs 1-3 are still unclear in gastric malignant tissues, and current tools are insufficient to rapidly quantify the expressional levels of HNPs 1-3 in tissues contrast to time-consuming immunohistochemistry. Moreover, we tried to investigate the binding capacity of HNPs-1 with GC cells in this study applied by a direct binding assay, followed by observations with MALDI-TOF MS and fluorescent microscopy. Further, the cytotoxic effect was also investigated using WST-1 assay.

MALDI-imaging mass spectrometry (IMS), a sophisticated, novel analytical tool, is designed to visualize the spatial distribution and determine the molecular composition of putative biomarkers [11,15-18]. MALDI-IMS is well adapted for studying smallmolecular-weight peptides or proteins ranged up to mostly $30 \mathrm{kDa}$, and the information can then be directly correlated to tissue histology [16]. Moreover, MALDI-IMS is useful for diagnosis and prognosis of patients or defining key proteins from needle or resected biopsies [16], and enable the combination of identification and localization for biological components directly from tissues without the need for additional techniques, such as radioactive or fluorescent regents [19]. Therefore, MALDI-TOF MS and derived IMS were used here to detect the expressions of HNPs 1-3.

In this study, we hypothesized that HNPs-1, -2 , and -3 are correlated with each other and overexpressed in GC tissues. Moreover, the increased level of HNPs 1-3 also implies the elevation of infiltrated neutrophils. The aim of this study was to evaluate the overexpression of HNPs 1-3 and disclose the spatial distributions of HNPs 1-3 by MALDI-IMS in GC. Moreover, we also investigated whether neutrophils accumulate in gastric tumor. On the other hand, the MALDI-TOF MS detection assay may complement existing diagnostic tools by enhancing the accuracy due to its highly sensitive and specific characters. Moreover, the determined binding capacity of HNPs- 1 could be further applied to in vivo imaging diagnostic and therapeutic probes by conjugating it with a chemotherapeutic component or radioactive isotope. 


\section{Materials and methods}

\subsection{Clinical tissue preparation}

The project was approved by the institutional review board of Taipei Medical University (no. P970302). Every matched-pair sample including tumor tissues and adjacent normal controls from individual patients was obtained by surgery, instantly frozen in liquid nitrogen, and stored at $-80^{\circ} \mathrm{C}$ for further investigation. Each pair of $10 \mu \mathrm{m}$-thick tissue sections was obtained on a cryostat (Microm HM525, Thermo Scientific) at $-20^{\circ} \mathrm{C}$ and deposited on indium tin oxide (ITO) coated glass slides (Delta Technologies) for the MALDIIMS analysis or on PEN membrane glass slides for laser capture microdissection (Arcturus ${ }^{\mathrm{XT}}$ ). Subsequently consistently cut tissues were also acquired and attached on to general glass slides for methylene blue staining, immunohistochemistry, and naphthol AS-D (3-Hydroxy-2-naphthoic-o-toluidide) chloroacetate esterase staining. Tumor grades were determined according to the American Joint Commission on Cancer Staging (AJCCS) system by an experienced pathologist under microscopy (BX51, Olympus) after staining with $0.1 \%$ methylene blue for $1 \mathrm{~min}$.

\subsection{Matrix spotting}

For the MALDI-IMS analysis, one tissue sample with stage IV and its adjacent normal control, both checked by methylene blue staining (Fig. 1A), were selected to be spotted with the fresh sinapinic acid (SA) matrix. An automatic sprayer (Chemical Inkjet Printing, ChIP1000, Shimadzu Biotech) was used to deliver a 100-picoliter volume of matrix solution $(20 \mathrm{mg} / \mathrm{ml}$ SA, $50 \%$ acetonitrile, and $0.1 \%$ trifluoroacetic acid in deionized water) onto the tissue surfaces using piezoelectric technology. The distance between the drops was set to $150 \mu \mathrm{m}$, and the total quantity of solution was set to 7500 picoliter by spraying 3 drops of 100 picoliter matrix solution each time. To obtain separate crystal spots on the tissue, it was necessary to wait 60,000 msec between cycles.

\subsection{MALDI-TOF MS}

The MALDI-TOF MS instrument (UltraFlexIII, Bruker Daltonics) was used to enforce the main pro- cessing with a standard 337-nm N $\mathrm{N}_{2}$ laser at $1000 \mathrm{~Hz}$ and a laser spot size of $50 \mu \mathrm{m}$. The instrument was operated in a positive linear mode at $26 \% \sim 28 \%$ laser power. In total, 1200 laser shots were accumulated from each deposited matrix droplet. The spectral data were base line-subtracted using Flexanalysis software and then rendered to the ClinProt platform for statistical analysis to pick the differential peaks in the MALDI-IMS analysis. The data calculated by ClinProt software was rendered to the Flexanalysis platform again for image visualization.

\subsection{Immunohistochemistry}

Immunohistochemistry was performed using a standard peroxidase-based staining method. Fresh tissue sections $(10 \mu \mathrm{m})$ were dried on a heater at $37^{\circ} \mathrm{C}$ and consecutively immersed in sequential $75 \%, 95 \%$, and $100 \%$ ethanol for $1 \mathrm{~min}$ for protein fixation. Briefly, endogenous peroxidase activity was quenched with $3 \%$ hydrogen peroxide for $10 \mathrm{~min}$. The antigen epitope was exposed by immersing the tissue slide in $10 \mathrm{mM}$ citrate acid within boiling water for $25 \mathrm{~min}$. Successive incubations with the primary goat anti-HNPs-1 antibody (PeproTech, 091CY133G) and the horseradish peroxidase (HRP)-conjugated anti-goat antibody were performed for $1 \mathrm{~h}$ in phosphate-buffered saline (PBS) buffer with shaking. An AEC kit (Sigma) was used for staining, following by the attached manual. Briefly, a mixed solution of $2.5 \mathrm{M}$ of acetate buffer, AEC chromogen (3-amino-9ethylcarbazole) and 3\% hydrogen peroxide were performed instantly and consecutively incubated with tissue slides. The image of stained slides was observed and acquired under microscopy (BX51, Olympus).

\subsection{Intensity fading $M S$}

AGS cells were cultured in $10 \mathrm{ml}$ of F12K medium, which included $10 \%$ fetal bovine serum and $1 \%$ antibiotic (a mixture of $100 \mathrm{U} / \mathrm{ml}$ of penicillin and $100 \mu \mathrm{g} / \mathrm{ml}$ streptomycin), within a $5 \% \mathrm{CO}_{2}, 37^{\circ} \mathrm{C}$ incubator for $48 \mathrm{~h}$. AGS cells were suspended by trypsin treatment and collected by 1000 -rpm centrifugation for $10 \mathrm{~min}$. A series of AGS cells (cell numbers of $5 \times 10^{3}, 10^{4}$, and $2 \times 10^{4}$ ) were incubated with $1 \mu \mathrm{g}$ of HNPs- 1 for $1 \mathrm{~h}$. Each mixture was centrifuged at $1000 \mathrm{rpm}$ to separate the supernatants and pellets. Supernatants at $1 \mu \mathrm{l}$ were mixed with $20 \mathrm{mg} / \mathrm{ml} \mathrm{SA}$ at a 1:1 ratio and spotted onto a steel plate until completely dry for further analysis. On the other hand, the pellets (AGS cells) were 
A
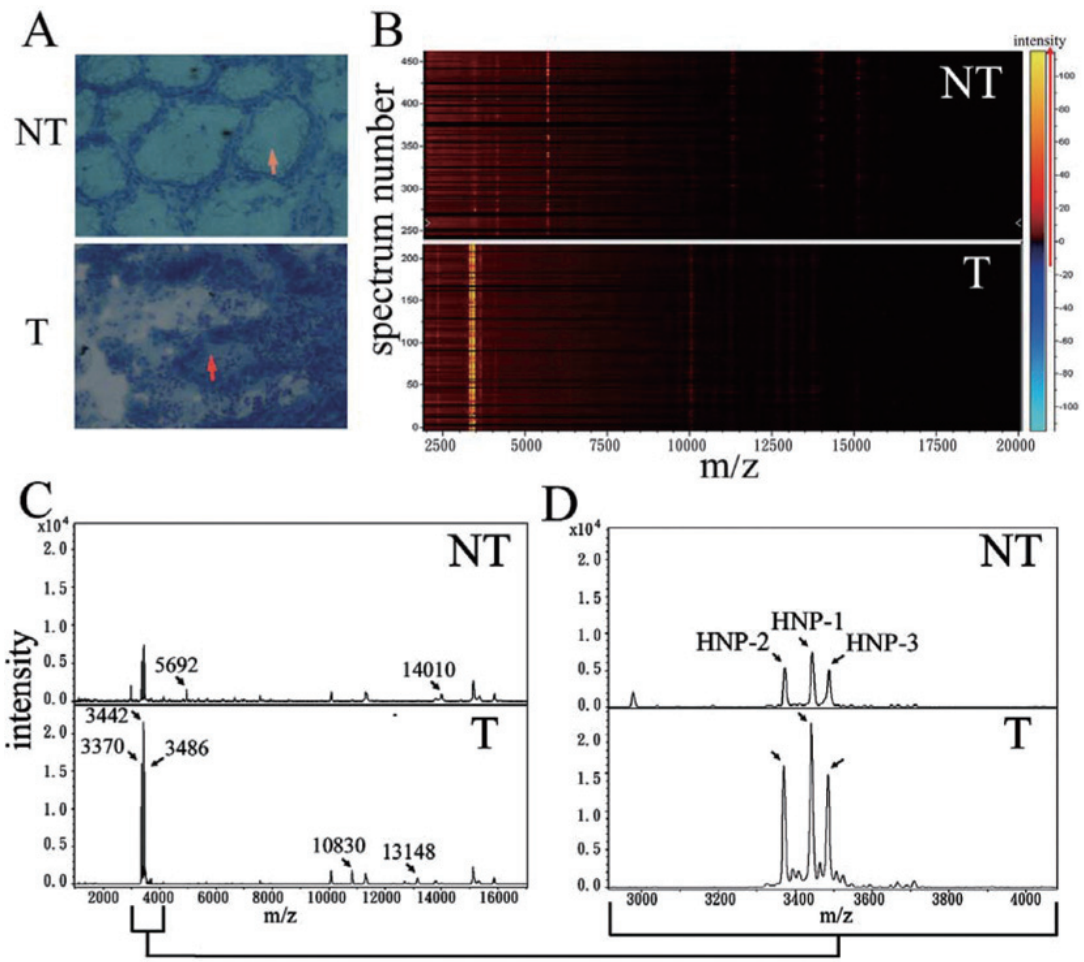

$\mathrm{D}$

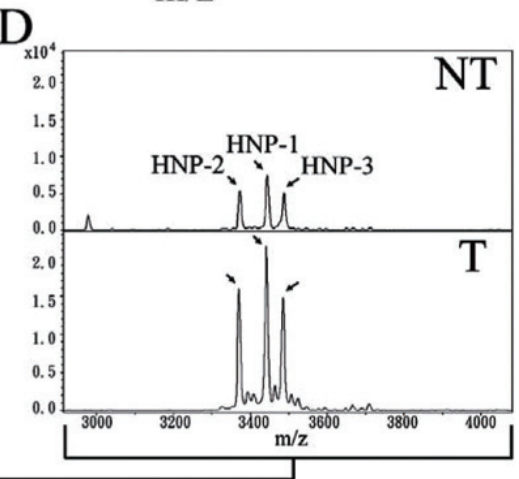

Fig. 1. Detection of HNPs-1, -2 and -3 using the MALDI-TOF MS analytical technique. (A) Histopathological confirmation of both cancerous tumor (T) and non-tumor (NT) tissues of each gastric specimen was carried out by staining with methylene blue. (B) An overview of the protein distribution within a virtual gel view of the MALDI-TOF MS analysis, which generated by obtained spectra of normal (220 spots) and tumor (460 spots) with a laser spot size of $50 \mu \mathrm{m}$ on tissues. (C) Differential peaks on the spectral protein profile between T and NT tissues. Two peaks (m/z 5692 and 14,010) were present in the normal region of gastric specimens and two defined peaks (m/z 10,830 and 13,148) specifically existed in the cancerous region. Furthermore, three peaks (m/z 3370, 3442, and 3486) showed significantly higher intensity in the cancerous region, compared to the normal region. Each peak was labeled with a black arrow (D) The detailed spectra of the peaks at m/z 3370,3442 , and 3486 were determined to be HNPs-1, -2 and -3 , respectively.

washed with PBS buffer three times, directly pipetted onto ITO slides, and transferred to a heater at $37^{\circ} \mathrm{C}$ until completely dry. Each $1 \mu \mathrm{l}$ of $20 \mathrm{mg} / \mathrm{ml}$ of SA was pipetted onto every plaque of cells three times to acquire adequate protein-matrix crystals and to extract sufficient tissue proteins on the surface. The operating conditions of MALDI-TOF MS were the same as those of MALDI-IMS previously described.

\subsection{In vitro binding assay}

AGS cells were cultured as previously described to acquire an adequate amount of cells in an eight-well chamber slide, subsequently fixed on a heater at $37^{\circ} \mathrm{C}$, and immersed in an ethanol series at $75 \%, 90 \%$, and $100 \%$ individually for $1 \mathrm{~min}$. Fixed cells were incubated with $50 \mu \mathrm{g} / \mathrm{ml}$ of HNPs-1 for $1 \mathrm{~h}$. The goat anti-HNPs-1 antibody and fluorescein isothiocyanate (FITC)-conjugated anti-goat antibody were then sequentially added and reacted for $1 \mathrm{~h}$. Between each reaction procedure, washing by PBS buffer was necessary. Images were acquired under visible white light and adequate fluorescent wavelength (excitation: $490 \mathrm{~nm}$; emission: $520 \mathrm{~nm}$ ) of microscopy (BX51, Olympus). Meanwhile, the control was processed by the same procedure except HNPs-1 incubation.

\subsection{WST-1 assay}

The WST-1 (2-(4-iodophenyl)-3-(4-nitrophenyl)-5(2,4-disulfophenyl)-2H-tetrazolium, monosodium salt) (Takara) assay was used to observe the cytotoxicity of HNPs-1 and cell proliferation after incubation for $48 \mathrm{~h}$. The detection processes followed the attached instructions. Each well of a 96-well microplate contained 2 $\times 10^{4}$ cells in $200 \mu \mathrm{l}$ of culture medium, and there were at least six replicates. A dose-dependent manner was performed and the concentrations of added HNPs1 were 10,50 , and $100 \mu \mathrm{g} / \mathrm{ml}$. Meanwhile, the positive 
and negative controls used $2 \%$ Triton X-100 and no addition, respectively.

\subsection{Statistical analysis}

The statistical software SPSS was used to calculate the significance according to Student's $t$-test. The significance ( $p$ value) was acceptable at $<0.05$. The coefficient of variation $(\mathrm{CV})$ was defined as the standard deviation to mean.

\section{Results}

\subsection{Detection of HNPs 1-3 by MALDI-IMS}

First, we intended to detect the expressions and distributions of HNPs 1-3 using MALDI-IMS. Each pair of GC tissues from the same patients was stained by methylene to individually determine normal and cancerous tissues (Fig. 1A). MALDI-TOF MS-derived technology, MALDI-IMS, was used to detect the protein signal and also observe the spatial distributions and co-localizations of HNPs-1, -2 and -3 in clinical tissues. The detection mass spectrum ranged $2 \sim 20 \mathrm{kDa}$ due to the limitation of the low resolution in high-mass capture $(>20 \mathrm{kDa})$ with MALDI-TOF MS. A representative proteomic map is shown by a gel view of the mass spectrum in Fig. 1B. Two peaks (m/z 5692 and 14,010) and five peaks (m/z 3370, 3442, 3486, $10,830$ and 13,148$)$, indicated by arrows, individually increased or decreased in tumorous tissues compared to the normal controls (Fig. 1C). According to our aim, HNPs 1-3 were the major items observed in the experimental process. The results showed that HNPs $1-3(\mathrm{~m} / \mathrm{z}$ 3370, 3442 and 3486) were apparently overexpressed in GC tissues; by contrast, lower signals appeared in normal gastric tissues (Fig. 1C, D). Among the triad, HNPs-1 was always the major peak, and HNPs-3 was consistently the minor peak. This phenomenon was also reported by Lundy's group using a MALDI-IMS approach to detect the HNPs 1-3 in gingival crevicular fluid [20]. Furthermore, by selecting their specific molecular masses, the imaging data showed that HNPs 1-3 were co-locatlized on the same tissue positions (all $p<0.01$ in ClinProt, Fig. 2A).

\subsection{Verification of the overexpressions of HNPs 1-3}

We further investigated 33 other pairs of GC tissues (Table 1) to validate the expression of HNPs 1-3 and
Table 1

Characteristics of gastric cancer patients

\begin{tabular}{cccc}
\hline $\begin{array}{c}\text { Patient } \\
(n=33)\end{array}$ & $\begin{array}{c}\text { Gender } \\
\text { female/male (n) }\end{array}$ & Age (year) & $\begin{array}{c}\text { HNPs-1 } \\
\text { (T/NT: }>2 \text { fold) }\end{array}$ \\
\hline I/II: 6 & $1 / 5$ & $70 \pm 11$ & $1(17 \%)$ \\
III: 10 & $1 / 9$ & $68 \pm 14$ & $4(40 \%)$ \\
IV: 17 & $11 / 6$ & $61 \pm 15$ & $11(65 \%)$ \\
\hline
\end{tabular}

Stages were determined according to the rules of the AJCC (The American Joint Committee on Cancer). HNPs, human neutrophil peptides; T, tumor; NT, non-tumor.

observed correlations among HNPs- $1,-2$, and -3 using MALDI-TOF MS. MALDI-IMS proved useful for detecting the presence of HNPs 1-3. However, each analytical spectrum measured by MALDI-TOF MS instrument has a different intensity under the flexible conditions of laser strength and frequency. In this study, we first tested a cohort of standard peptides (angiotensin II (m/z 1046), angiotensin I (m/z 1296), substance P (m/z 1347), bombesin (m/z 1619), ACTH clip 1-17 (m/z 2096), ACTH clip 18-39 (m/z 2465), and somatostatin ( $\mathrm{m} / \mathrm{z} 3147)$, Bruker) of the same amount in different concentration solutions of HNPs- 1 at $0.0078 \sim$ $0.5 \mathrm{~g} / \mathrm{L}$ to ensure that using MALDI-TOF MS for the expression analysis was feasible. Subsequently, the ratio of $\mathrm{m} / \mathrm{z} 3442$ (HNPs-1) to m/z 3147 (somatostatin) was determined, and a standard curve was calculated and completed (Fig S1A and B, $r^{2}=0.98, \mathrm{CV}_{\text {inter }}=$ $18 \%$ and $\mathrm{CV}_{\text {intra }}=16 \%$ as HNPs- 1 at $0.5 \mathrm{~g} / \mathrm{L}$ ) which demonstrated that MALDI-TOF MS can be used for qualitative analysis of HNPs 1-3. On the other hand, in order to ensure the quantification of HNPs 1-3 by MALDI-TOF MS in the tissue of gastric cancer, we measured the levels of external HNPs- 1 at $0 \sim 1000$ $\mathrm{mg} / \mathrm{L}$ (Fig. S1C). The result showed that the linear dynamic range of HNPs-1 in real tissues can be three orders of magnitude (Fig. S1D, $r^{2}=0.99, \mathrm{CV}_{\text {inter }}=$ $11 \%$ and $\mathrm{CV}_{\text {intra }}=9 \%$ as HNPs-1 at $1000 \mathrm{mg} / \mathrm{L}$ ) from 0.07 to 4.33 of HNPs- 1 to sum of peaks, indicating quantification of HNPs-1 using MALDI-TOF MS was feasible due to the highest ratio in the stage IV of gastric cancer was $0.20 \pm 0.04$ (Fig. 2F).

MALDI-TOF MS results showed that HNPs 1-3 demonstrated significant differences in abundances in cancerous tissues compared to normal tissues (Fig. 2E) consistent with the investigation by MALDI-IMS. The $\mathrm{CV}_{\text {inter }}$ and $\mathrm{CV}_{\text {intra }}$ values of MALDI-TOF used in the clinical tissues analysis were $18 \%$ and $5 \%$, respectively. HNPs-1, -2 and -3 were positively correlated with each other (Fig. 2B-D, all $p<0.0001$ ), implying that HNPs 1-3 were deposited at the same position. Then we selected the major peak, HNPs-1, for further 

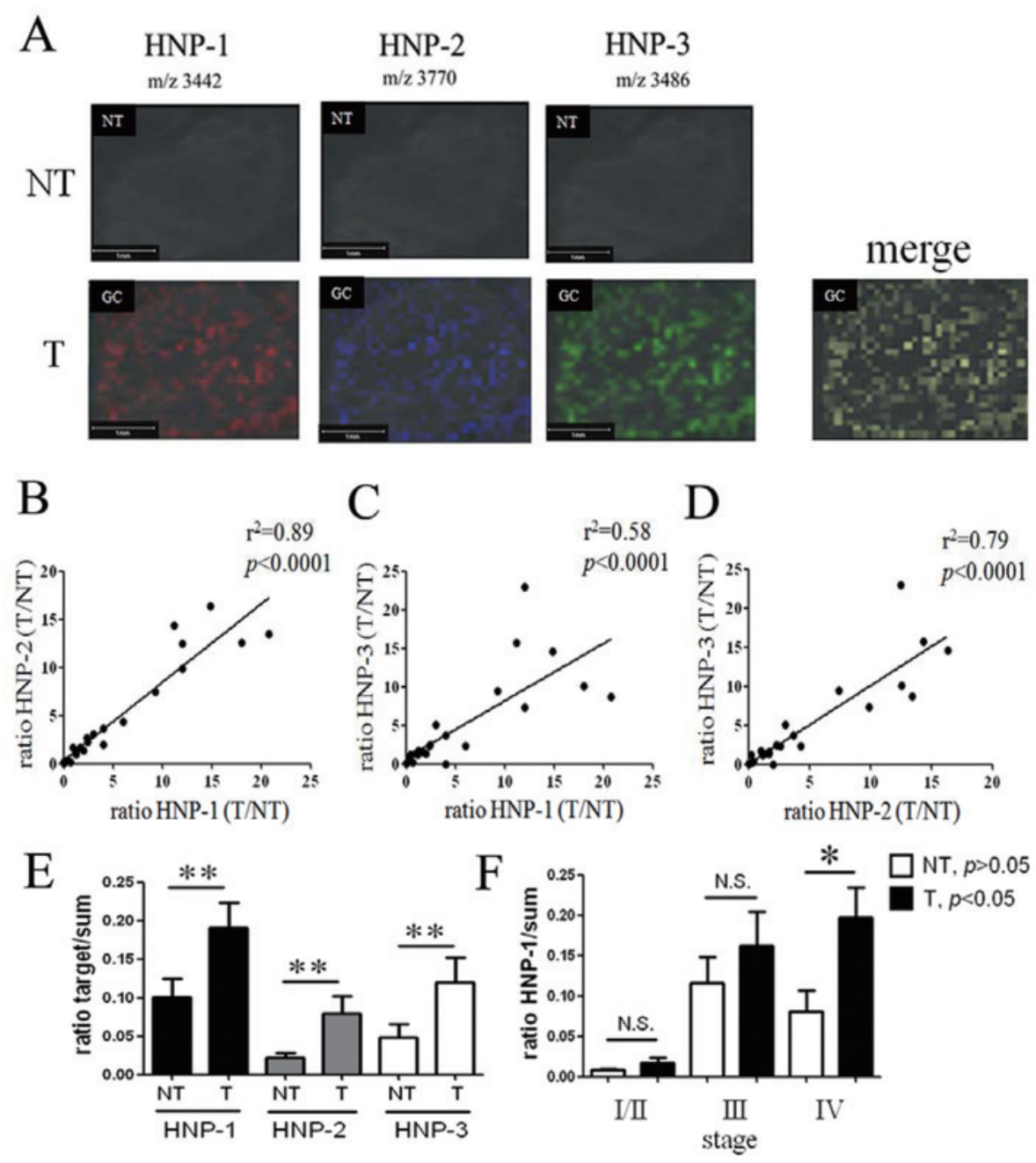

Fig. 2. Spatial contribution, correlation, and expressions of HNPs $1-3$. (A) HNPs-1 (red, m/z 3442), -2 (blue, m/z 3770 ), and -3 (green, m/z 3486) in the same cancerous tissue were determined by MALDI-IMS. The triad was significantly overexpressed in cancerous tissue compared to normal tissue. The triad overlapped at the same positions, whereas HNPs-1 was the major peak and was higher than the other two. The results imply that the triad may have the common biological functions in gastric cancer (GC) tissue. (B-D) Other pairs of GC tissues ( $n=33$ ) were measured to observe the expressions of HNPs-1, -2 , and -3 using MALDI-TOF MS direct analysis on tissue slides. HNPs-1, -2 and -3 were positively correlated with each other. (E) Semi-quantification by MALDI-TOF MS revealed that HNPs 1-3 were overexpressed in tumor compared to non-tumor. Target: HNPs-1, -2 , or -3 . (F) Moreover, we show that the major peptide, HNPs-1, gradually increased from the early to advanced stage of GC. HNPs-1 was particularly elevated and differentiated in the advanced stage. Sum: the sum of all peaks detected by MALDI-TOF MS. ${ }^{*} p<0.05,{ }^{* *} p<0.01$. Scale bar, $1 \mathrm{~mm}$.

investigation. We determined that the overexpressed HNPs-1 gradually increased from the early to advanced tumor stage not only in the proportion of tumors to non-tumors (Table 1) but also at the expression level $(p<0.05$, Fig. 2F).

Immunohistochemistry was also used to verify the expression of HNPs-1 in the GC tissues. One pair of GC tissues was stained with methylene blue (Fig. 3, upper panel) to determine the pathological condition before immunohistochemistry. Consequentially anoth- er pair of tissue sections was determined by immunohistochemistry to detect the expression and position of HNPs-1. The results demonstrated that HNPs-1 appeared in the cancerous tissue, and was upregulated compared to that in normal control, and abundantly presented in the lamina propria (Fig. 3B, middle panel). Next, we used laser capture microdissection to separate and obtain tissues of the gastric gland and lamina propria from cancerous tissues in order to confirm the expression of HNPs- 1 in the lamina propria, 


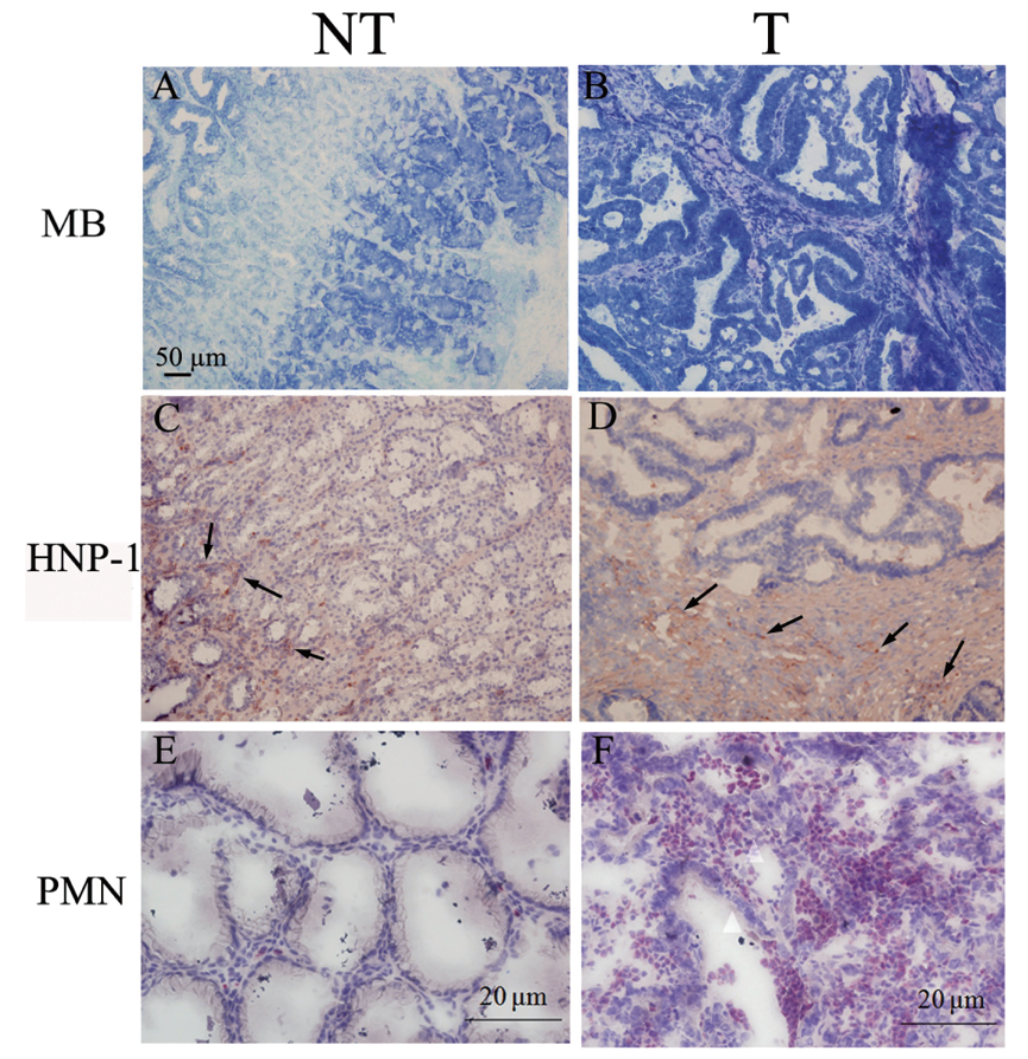

Fig. 3. Verification of HNPs-1 and neutrophil expressions. The expression of HNPs-1 was confirmed by immunohistochemistry. (upper panel) One pair of samples was stained with methylene blue to ensure the pathology. (middle panel) The same tissue slide was analyzed by immunohistochemistry. Results showed that HNPs-1 (indicated by arrows) was mainly located in the lamina propria of both adjacent normal and gastric cancer tissue. HNPs-1 appeared on cancerous tissue more than normal control. (lower panel) Infiltrated neutrophils were observed to have accumulated in tumor tissues detected by naphthol AS-D chloroacetate esterase staining. MB, methylene blue; PMN, polymorphonuclear neutrophils.

and immediately analyzed the captured sections using MALDI-TOF MS to observe the expression of HNPs1. The results revealed that HNPs-1 more abundantly appeared in the lamina propria than that in the gastric gland (Fig. S2B).

\subsection{Neutrophil accumulation in GC tissues}

We proposed that the increased HNPs 1-3 may direct the accumulation of infiltrated neutrophils in tumors. In order to observe the accumulation of neutrophils in GC tissues, we performed naphthol AS-D chloroacetate esterase staining. We observed that the infiltrated neutrophils accumulated in cancerous tissues more than that in normal tissue (Fig. 3, lower panel), supporting neutrophils specifically appearing in tumor tissues. The same pair of tissue samples was also investigated by MALDI-TOF MS, which demonstrated that neutrophil-secreted peptides, HNPs 1-3 (m/z 3370,
3442, and 3486), were overexpressed in tumor tissues more than in normal tissues, indicating that the overexpressions of HNPs 1-3 were correlated with elevated neutrophils.

\subsection{Role of HNPs-1 in GC}

In order to reveal the function of HNPs-1, an in vitro binding assay was used to determine the binding capacity of HNPs-1 with the GC cell line, AGS, including intensity-fading MS and an immunofluorescent assay. The intensity-fading MS assay was first designed to observe the protein-protein interactions, which showed that HNPs-1 could directly interact with AGS cells without being retained in the supernatant (Fig. 4A). Moreover, the spectrum intensity increased from a low to high amount of AGS cells, which indicated that only a sufficient accumulation of HNPs-1 could be detected by this approach. On the other hand, the control 
A

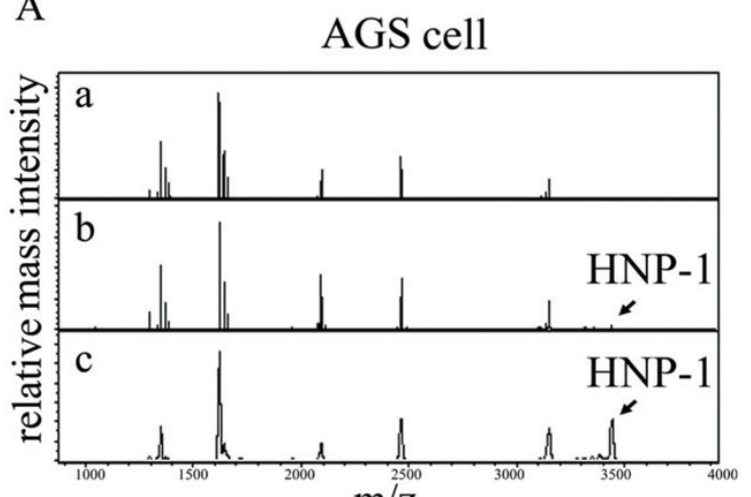

B

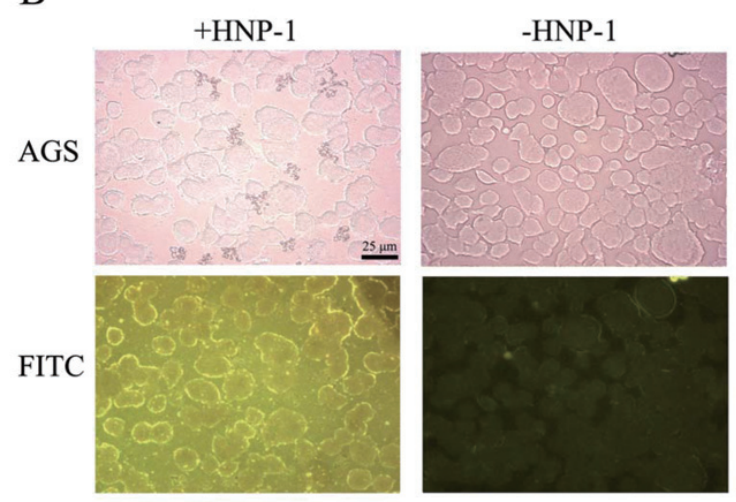

Fig. 4. Binding capacity of HNPs-1 to gastric cancer AGS cells. (A) A novel MALDI-TOF MS-derived technique, and intensity fading MS present the binding capacity of HNPs- 1 with AGS cells (a: $5 \mathrm{x}$ $10^{3}$; b: $10^{4}$; c: $2 \times 10^{4}$ cells). Cells bound with $1 \mu \mathrm{g}$ of HNPs- 1 were analyzed by MALDI-TOF MS as directly conducted on a steel plate. The results demonstrate that HNPs- 1 has the capacity to bind to AGS cells. (B) Observation of external HNPs-1 binding to AGS cells by an immunofluorescent assay. Upper two panels: the cell distribution with or without HNPs-1 incubation under visible white light. Lower two panels: a clump of AGS cells incubated with HNPs-1 show higher fluorescent signals on the cell surface, moreover, the control without adding HNPs-1 contrarily presented no fluorescence. Scale bar, $25 \mu \mathrm{m}$.

without adding HNPs-1 for binding could not detect the HNPs-1 signal. The result demonstrates that AGS cells do not secrete HNPs-1 or only secrete an extremely small amount of HNPs-1 which is insufficient for detection by MALDI-TOF MS. Next, an in vitro binding assay in tandem with an immunofluorescent assay allowed us to directly observe the binding phenomenon under a microscope. This result indicated that the fluorescent signal obviously appeared on the surface of AGS cells more than the control to which no external HNPs-1 was added (Fig. 4B). Moreover, the control image also demonstrated that AGS cancer cells did not directly secrete HNPs-1.
A

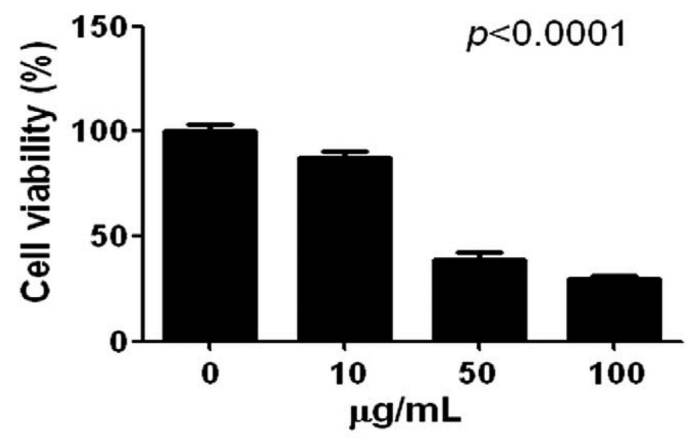

B

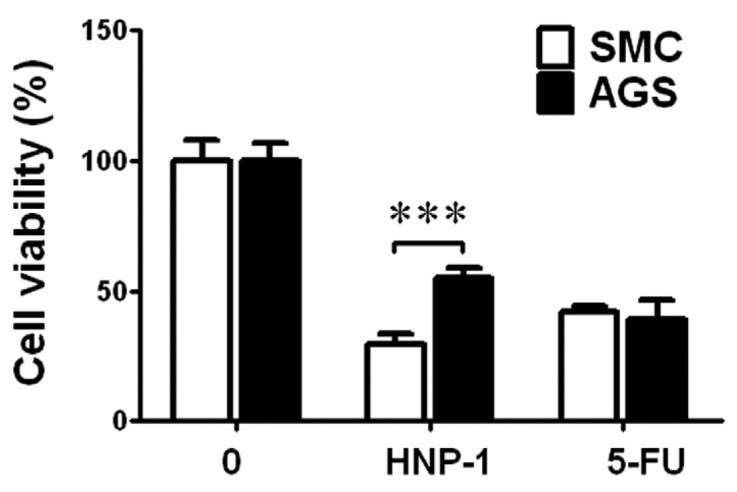

Fig. 5. Cytotoxic effect of HNPs-1 on AGS cells. Three concentrations of HNPs-1, 10, 50 and $100 \mu \mathrm{g} / \mathrm{ml}$, were added to the culture medium. (A) A WST-1 assay was used to observe the cell number after treatment with HNPs-1. Results showed that adding over $50 \mu \mathrm{g} / \mathrm{ml}$ of HNPs-1 could dramatically inhibit AGS cell growth. Meanwhile, $2 \%$ Triton X-100 were used as positive control and nothing was added to the negative controls. In addition, an unrelated peptide was also used as a control for the WST-1 assay. (B) Gastric smooth muscle cells were used as a normal control to specify the cytotoxicity of HNPs-1 toward AGS cells. Meanwhile, $200 \mu \mathrm{M}$ of 5-FU was added to both AGS cells and gastric smooth muscle cells (SMCs) to validate the specific inhibition of HNPs-1 for AGS cells. $* * p<0.01$, *** $p<0.001$.

By further investigating whether the antibacterial peptide, HNPs-1, has the same function in tumor cells, the inhibitory capacity of HNPs-1 was investigated in a dose dependent manner. Figure 5A shows that HNPs-1 inhibited AGS cell growth in vitro in a dose-dependent manner, and lysed some tumor cells (data not shown), indicating that HNPs-1 had a cytotoxic effect on AGS cells. Moreover, we specified the inhibitory ability of HNPs-1 to AGS cells. Normal cell, gastric smooth muscle cells (SMCs), were used, and $100 \mu \mathrm{g} / \mathrm{ml}$ of HNPs-1 caused minimal effects on SMCs compared to AGS cells (Fig. 5B), revealing that HNPs-1 exhibited specific inhibition toward AGS cells. 


\section{Discussion}

This study identifies the expressions of HNPs 1-3 in GC tissues on the same position correlated with each other as upregulated biomarkers; moreover, elevated neutrophils were also observed. To our knowledge, HNPs 1-3 are single-chain cationic peptides that were discovered to play critical roles in polymorphonuclear leukocytes as antimicrobial components. A recent report showed that HNPs 1-3 are overexpressed in many solid tumors as cancer biomarkers, and also have the potential to trigger cell death as antitumor molecules [12]. In this study, we verified that the HNPs 1-3 were correlated and had the potential to serve as diagnostic tissue targets in GC patients. Moreover, the overlapping character implies that this triad has common biological functions in cancerous tissues and indicates the elevation of infiltrated neutrophils.

A previous study have showed that HNPs 1-3 are significantly increased in gastric fluid of GC patients [13]. On the other hand, Isomoto and coworkers indicated that HNPs 1-3 were associated with Helicobacter pylori infection [21]. Therefore, HNPs 1-3 may increase due to elevated neutrophils which are recruited to infectious tissues. Chronic inflammation is associated with cancer development [22]. Recently, tumor-associated neutrophils were reported to participate in tumor promotion, progression, and metastasis [23,24]. Previous reports showed that a high neutrophil-lymphocyte ratio in the clinic can be used as a biomarker to predict poor prognoses of various cancers [21,25-27], indicating that the inflammatory situation in patients with a tumor may recruit neutrophils and lead to a poor prognosis. Herein, we demonstrated that neutrophils positively accumulated in GC tissues and may be used as a target for a diagnosis. Therefore, targeting neutrophils may be helpful for tumor diagnosis and therapy. A recent study showed that using a CXCR2-specific antagonist could reduce tumor growth in lung adenocarcinomas by blocking neutrophil recruitment [28]. Based on our research in this study, HNPs 1-3 combined with MALDITOF MS can be used to rapidly detect infiltrated neutrophils in biopsy specimens to determine a therapeutic strategy, such as using a neutrophil-specific inhibitor. Moreover, an inflammatory imaging agent, such as cFLFLFK-PEG-64Cu, used to target neutrophils can specifically be used for tumor imaging [29].

With detection of HNPs-1 by MALDI-TOF MS, we found that overexpressions of HNPs 1-3 were associated with the tumor stage (Table 1, Fig. 2F). The functions of HNPs 1-3 appearing in cancerous tissues ur- gently need to be revealed. Originally HNPs 1-3 were believed to play substantial roles in innate immunity against bacterial, fungal, and viral pathogens. In this study, we directly utilized an in vitro binding assay to discover the putative function of HNPs-1. We found that HNPs- 1 could bind to the surface of AGS cells, and influenced the growth of AGS cells in vitro. These primary results demonstrate that HNPs- 1 has the potential to serve as a targeting probe and an antitumor molecule. Although the cytotoxic effects were discovered in other cancers [30-32], this is the first study to demonstrate that HNPs-1 is associated with the cell growth of GC. The real functions of HNPs 1-3 stimulate deep thought. Lundy's group indicated that the upregulation of HNPs 1-3 may primarily be produced from tumor-infiltrating immune cells, including neutrophils [10]. In this study, we found that HNPs 1-3 were abundantly located in the lamina propria of cancerous tissues compared to the gastric gland, implying that neutrophils may assemble in GC tissues and secrete HNPs 1-3. HNPs-1 probably inhibits angiogenesis by affecting cell proliferation, adhesion, and migration [12]. In an in vivo study of nude mice with human lung adenocarcinoma xenografts, $\mathrm{Xu}$ and coworkers indicated that intracellular HNPs-1 induced tumor cell apoptosis and inhibited tumor growth [33]. In our case, we treated AGS cells by adding 50 or $100 \mu \mathrm{g} / \mathrm{ml}$ HNPs- 1 for $48 \mathrm{~h}$, and the results showed that both treatments inhibited cell growth. However, presently the multiple functions of HNPs-1-3 need to be revealed depending on the protein concentration as opposite functions to exert cytotoxic effects [34] or increase cell proliferation, motility and invasiveness [8]. Moreover, the mechanism by which GC cells accumulate and the receptor they interact with HNPs 1-3 are a matter of speculation.

\section{Conclusions}

Overexpressed HNPs 1-3 were found in the cancerous tissues in this present study, which directed elevated neutrophils. HNPs 1-3 are considered to be potentially significant biomarkers for the diagnosis and surveillance of GC. This study identified the co-localization and correlation of HNPs 1-3 with high abundance in the lamina propria of tumors, and determined the binding capacity with GC cells in vitro. Our results suggest that HNPs 1-3 and neutrophils can be used as diagnostic targets and even as therapeutic drugs to develop noninvasive diagnostic tools and improve the survival rate in GC patients. 


\section{Competing interests}

The authors declare that they have no competing interests.

\section{Authors' contributions}

Chun-Chia Cheng: article writing and experimental operations; Shui-Cheng Lee: experimental design; Jungshan Chang: article revision and experimental design; Ling-Yun Chen: article revision. Fu-Der Mai and Chun-Chao Chang: helped with sample diagnosis and experimental design.

\section{Acknowledgements}

This project was supported by grant ARA010203 from the Atomic Energy Council of Taiwan, R.O.C.

\section{Supplemental material}

Supplementary data can be found at http://my2.tmu. edu.tw/sysdata/94/4194/doc/977f8d2ffdb37f14/attach/ 343247.pdf.

\section{References}

[1] L. Yang, Incidence and mortality of gastric cancer in China, World J Gastroenterol 12 (2006), 17-20.

[2] C.J. Chen, S.L. You, L.H. Lin, W.L. Hsu and Y.W. Yang, Cancer epidemiology and control in Taiwan: a brief review, Jpn J Clin Oncol 32 Suppl (2002), S66-S81.

[3] M. McCracken, M. Olsen, M.S. Chen, Jr., A. Jemal, M. Thun, V. Cokkinides, D. Deapen and E. Ward, Cancer incidence, mortality, and associated risk factors among Asian Americans of Chinese, Filipino, Vietnamese, Korean, and Japanese ethnicities, CA Cancer J Clin 57 (2007), 190-205.

[4] D.M. Parkin, F. Bray, J. Ferlay and P. Pisani, Global cancer statistics, 2002, CA Cancer J Clin 55 (2005), 74-108.

[5] A. Amin, H. Gilmour, L. Graham, S. Paterson-Brown, J. Terrace and T.J. Crofts, Gastric adenocarcinoma missed at endoscopy, J R Coll Surg Edinb 47 (2002), 681-684.

[6] J. Albrethsen, R. Bogebo, S. Gammeltoft, J. Olsen, B. Winther and H. Raskov, Upregulated expression of human neutrophil peptides 1, 2 and 3 (HNP 1-3) in colon cancer serum and tumours: a biomarker study, BMC Cancer 5 (2005), 8.

[7] J. Albrethsen, C.H. Moller, J. Olsen, H. Raskov and S. Gammeltoft, Human neutrophil peptides 1, 2 and 3 are biochemical markers for metastatic colorectal cancer, Eur J Cancer 42 (2006), 3057-3064.

[8] D.A. Holterman, J.I. Diaz, P.F. Blackmore, J.W. Davis, P.F. Schellhammer, A. Corica, O.J. Semmes and A. Vlahou, Overexpression of alpha-defensin is associated with bladder cancer invasiveness, Urol Oncol 24 (2006), 97-108.
[9] C.A. Muller, J. Markovic-Lipkovski, T. Klatt, J. Gamper, G. Schwarz, H. Beck, M. Deeg, H. Kalbacher, S. Widmann, J.T. Wessels et al., Human alpha-defensins HNPs-1, -2 , and -3 in renal cell carcinoma: influences on tumor cell proliferation, Am J Pathol 160 (2002), 1311-1324.

[10] F.T. Lundy, D.F. Orr, J.R. Gallagher, P. Maxwell, C. Shaw, S.S. Napier, C. Gerald Cowan, P.J. Lamey and J.J. Marley, Identification and overexpression of human neutrophil alphadefensins (human neutrophil peptides 1,2 and 3) in squamous cell carcinomas of the human tongue, Oral Oncol 40 (2004), 139-144.

[11] J.A. Bauer, A.B. Chakravarthy, J.M. Rosenbluth, D. Mi, E.H. Seeley, N. De Matos Granja-Ingram, M.G. Olivares, M.C. Kelley, I.A. Mayer, I.M. Meszoely et al., Identification of markers of taxane sensitivity using proteomic and genomic analyses of breast tumors from patients receiving neoadjuvant paclitaxel and radiation, Clin Cancer Res 16 (2010), 681-690.

[12] N. Droin, J.B. Hendra, P. Ducoroy and E. Solary, Human defensins as cancer biomarkers and antitumour molecules, $J$ Proteomics 72 (2009), 918-927.

[13] O.L. Kon, T.T. Yip, M.F. Ho, W.H. Chan, W.K. Wong, S.Y. Tan, W.H. Ng, S.Y. Kam, A. Eng, P. Ho, et al, The distinctive gastric fluid proteome in gastric cancer reveals a multibiomarker diagnostic profile, BMC Med Genomics 1 (2008), 54

[14] Y. Mohri, T. Mohri, W. Wei, Y.J. Qi, A. Martin, C. Miki, M. Kusunoki, D.G. Ward and P.J. Johnson, Identification of macrophage migration inhibitory factor and human neutrophil peptides 1-3 as potential biomarkers for gastric cancer, $\mathrm{Br} J$ Cancer 101 (2009), 295-302.

[15] H.K. Kim, M.L. Reyzer, I.J. Choi, C.G. Kim, H.S. Kim, A. Oshima, O. Chertov, S. Colantonio, R.J. Fisher, J.L. Allen, et al, Gastric cancer-specific protein profile identified using endoscopic biopsy samples via MALDI mass spectrometry, $J$ Proteome Res 9 (2010), 4123-4130.

[16] P. Chaurand, J.L. Norris, D.S. Cornett, J.A. Mobley, R.M. Caprioli, New developments in profiling and imaging of proteins from tissue sections by MALDI mass spectrometry, $J$ Proteome Res 5 (2006), 2889-2900.

[17] H. Meistermann, J.L. Norris, H.R. Aerni, D.S. Cornett, A. Friedlein, A.R. Erskine, A. Augustin, M.C. De Vera Mudry, S. Ruepp, L. Suter, et al, Biomarker discovery by imaging mass spectrometry: transthyretin is a biomarker for gentamicininduced nephrotoxicity in rat, Mol Cell Proteomics 5 (2006), 1876-1886.

[18] P. Chaurand, S. Fouchecourt, B.B. DaGue, B.J. Xu, M.L. Reyzer, M.C. Orgebin-Crist and R.M. Caprioli, Profiling and imaging proteins in the mouse epididymis by imaging mass spectrometry, Proteomics 3 (2003), 2221-2239.

[19] B.K. Kaletas, I.M. van der Wiel, J. Stauber, C. Guzel, J.M. Kros, T.M. Luider, R.M. Heeren, Sample preparation issues for tissue imaging by imaging MS, Proteomics 9 (2009), 26222633.

[20] F.T. Lundy, D.F. Orr, C. Shaw, P.J. Lamey and G.J. Linden, Detection of individual human neutrophil alpha-defensins (human neutrophil peptides 1, 2 and 3) in unfractionated gingival crevicular fluid-a MALDI-MS approach, Mol Immunol 42 (2005), 575-579.

[21] H. Isomoto, H. Mukae, H. Ishimoto, Y. Date, Y. Nishi, K. Inoue, A. Wada, T. Hirayama, M. Nakazato, S. Kohno, Elevated concentrations of alpha-defensins in gastric juice of patients with Helicobacter pylori infection, Am J Gastroenterol 99 (2004), 1916-1923. 
[22] A. Mantovani, P. Allavena, A. Sica and F. Balkwill, Cancerrelated inflammation, Nature 454 (2008), 436-444.

$23]$ Z.G. Fridlender, J. Sun, S. Kim, V. Kapoor, G. Cheng, L. Ling, G.S. Worthen and S.M. Albelda, Polarization of tumorassociated neutrophil phenotype by TGF-beta: "N1" versus "N2" TAN, Cancer Cell 16 (2009), 183-194.

[24] P.M. Hofman, Pathobiology of the neutrophil-intestinal epithelial cell interaction: role in carcinogenesis, World J Gastroenterol 16 (2010), 5790-5800.

[25] H. Shimada, N. Takiguchi, O. Kainuma, H. Soda, A. Ikeda, A. Cho, A. Miyazaki, H. Gunji, H. Yamamoto and M. Nagata, High preoperative neutrophil-lymphocyte ratio predicts poor survival in patients with gastric cancer, Gastric Cancer 13 (2010), 170-176.

[26] H. Cho, H.W. Hur, S.W. Kim, S.H. Kim, J.H. Kim, Y.T. Kim, $\mathrm{K}$. Lee, Pre-treatment neutrophil to lymphocyte ratio is elevated in epithelial ovarian cancer and predicts survival after treatment, Cancer Immunol Immunother 58 (2009), 15-23.

[27] H. Cho and J.H. Kim, Multiplication of neutrophil and monocyte counts (MNM) as an easily obtainable tumour marker for cervical cancer, Biomarkers 14 (2009), 161-170.

[28] S. Tazzyman, S.T. Barry, S. Ashton, P. Wood, D. Blakey, C.E. Lewis and C. Murdoch, Inhibition of neutrophil infiltration into A549 lung tumors in vitro and in vivo using a CXCR2specific antagonist is associated with reduced tumor growth, Int J Cancer (2011)

[29] L.W. Locke, M.D. Chordia, Y. Zhang, B. Kundu, D. Kennedy,
J. Landseadel, L. Xiao, K.D. Fairchild, S.S. Berr, J. Linden and D. Pan, A novel neutrophil-specific PET imaging agent: cFLFLFK-PEG-64Cu, J Nucl Med 50 (2009), 790-797.

[30] J.S. Mader and D.W. Hoskin, Cationic antimicrobial peptides as novel cytotoxic agents for cancer treatment, Expert Opin Investig Drugs 15 (2006), 933-946.

[31] S.T. McKeown, F.T. Lundy, J. Nelson, D. Lockhart, C.R. Irwin, C.G. Cowan and J.J. Marley, The cytotoxic effects of human neutrophil peptide-1 (HNP1) and lactoferrin on oral squamous cell carcinoma (OSCC) in vitro, Oral Oncol 42 (2006), 685-690.

[32] S. Ghavami, A. Asoodeh, T. Klonisch, A.J. Halayko, K. Kadkhoda, T.J. Kroczak, S.B. Gibson, E.P. Booy, H. NaderiManesh and M. Los, Brevinin-2R(1) semi-selectively kills cancer cells by a distinct mechanism, which involves the lysosomal-mitochondrial death pathway, J Cell Mol Med 12 (2008), 1005-1022.

[33] N. Xu, Y.S. Wang, W.B. Pan, B. Xiao, Y.J. Wen, X.C. Chen, L.J. Chen, H.X. Deng, J. You, B. Kan, et al, Human alphadefensin-1 inhibits growth of human lung adenocarcinoma xenograft in nude mice, Mol Cancer Ther 7 (2008), 15881597.

[34] J. Aarbiou, G.S. Tjabringa, R.M. Verhoosel, D.K. Ninaber, S.R. White, L.T. Peltenburg, K.F. Rabe and P.S. Hiemstra, Mechanisms of cell death induced by the neutrophil antimicrobial peptides alpha-defensins and LL-37, Inflamm Res 55 (2006), 119-127. 


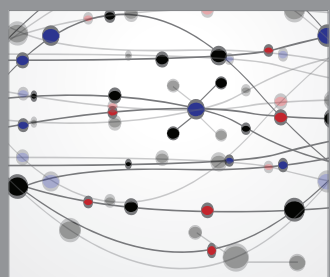

The Scientific World Journal
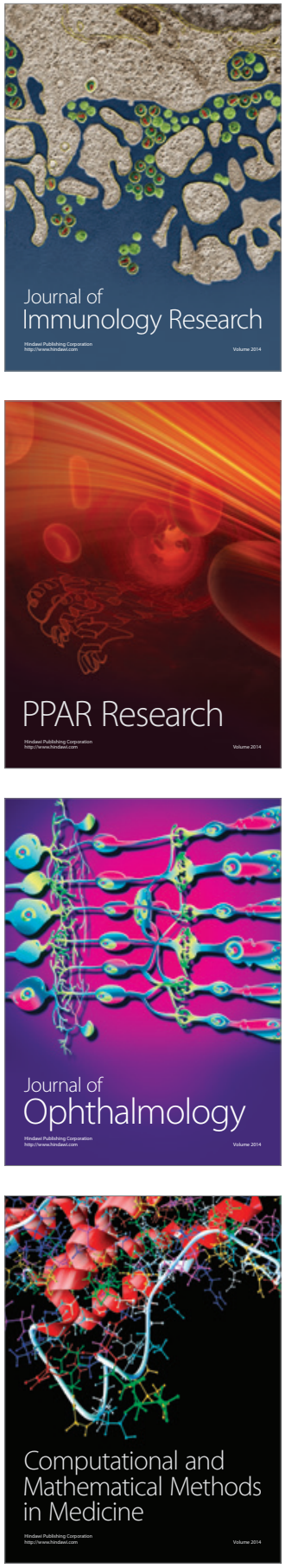

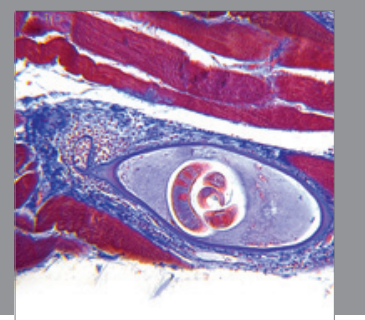

Gastroenterology

Research and Practice
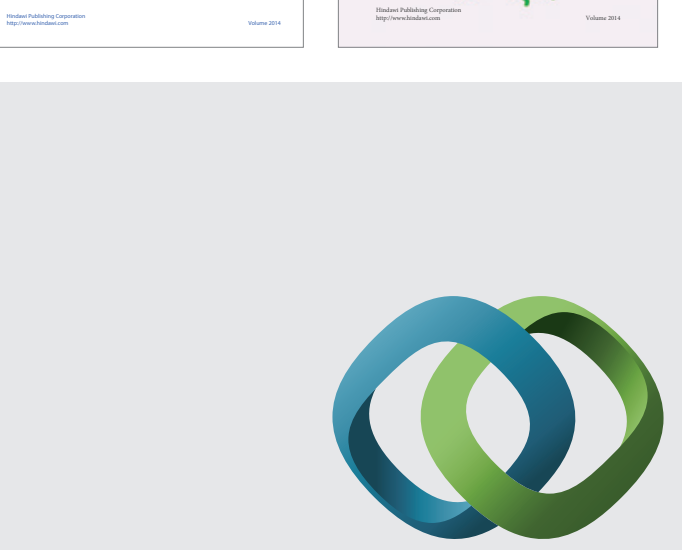

\section{Hindawi}

Submit your manuscripts at

http://www.hindawi.com
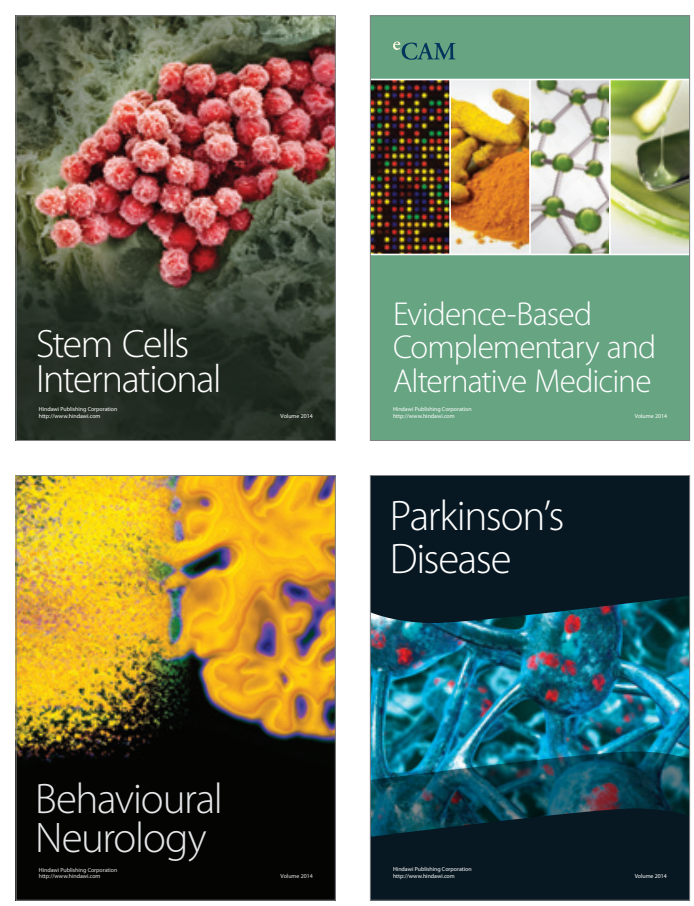

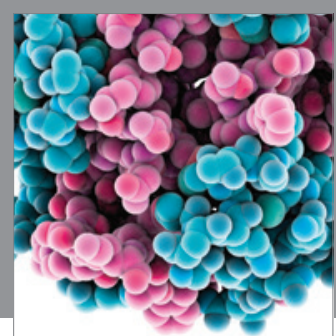

Journal of
Diabetes Research

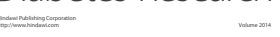

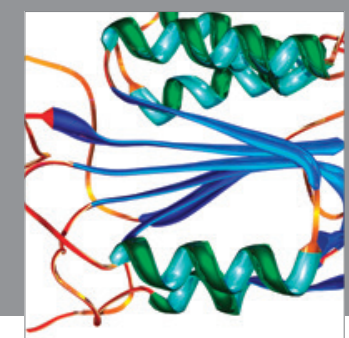

Disease Markers
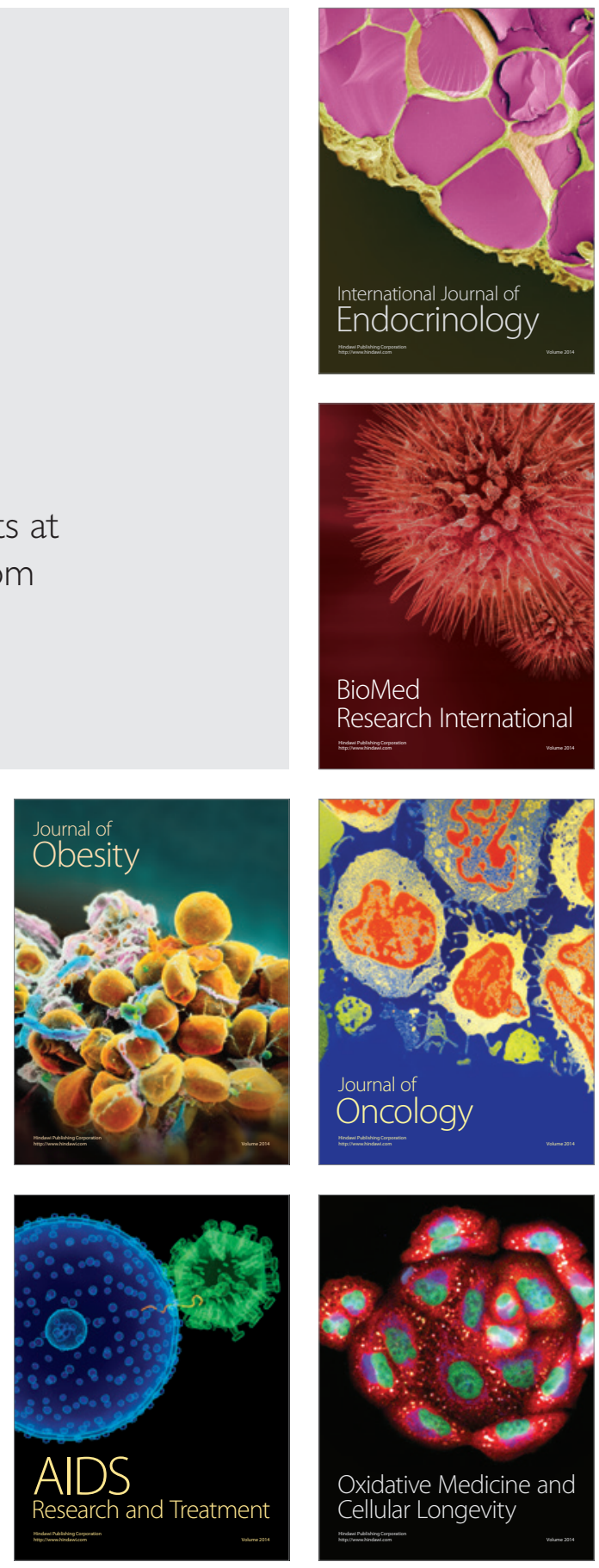